\title{
Limb Congestion and Sympathoexcitation during Exercise Implications for Congestive Heart Failure
}

Joseph McClain, Cullen Hardy, Belinda Enders, * Michael Smith, * and Lawrence Sinoway

Division of Cardiology and the *Department of Radiology, The Milton S. Hershey Medical Center, The Pennsylvania State University, Hershey, Pennsylvania 17033

\begin{abstract}
During static exercise, heart failure (HF) subjects activate the sympathetic nervous system differently than normal controls. HF causes metaboreceptor desensitization with either enhanced mechanoreceptor activity or central command. In this report, we examined whether increased muscle interstitial pressure, as seen in HF, augments other neural systems. We measured muscle sympathetic nerve activity (MSNA; peroneal nerve) in 10 normals during static exercise (40\% maximal voluntary grip) and posthandgrip circulatory arrest (PHG-CA). This was repeated after venous congestion ( VC; cuff inflation to $90 \mathrm{mmHg}$ ). VC increased forearm volume (plethysmography) by $4.7 \%$. MSNA responses to exercise were greater after VC (150.5 \pm 41.8 vs. $317.3 \pm 69.9$ arbitrary units; $P<0.01$ ). However, MSNA responses during PHG-CA were not affected by $\mathrm{VC}$, and ${ }^{31} \mathrm{P}$ nuclear magnetic resonance $(n=5)$ demonstrated no effect of $\mathrm{VC}$ on $\mathrm{pH}$ or $\mathrm{H}_{2} \mathrm{PO}_{4}^{-}$. Similar effects of $\mathrm{VC}$ on MSNA were noted after ischemic exercise $(n=7)$, excluding flow alterations as the explanation. VC probably sensitized mechanically sensitive afferents since MSNA during involuntary biceps contractions increased after VC $(n=6)$, and skin sympathetic nerve responses during handgrip, an index of central command, were not increased by VC $(n=6)$. (J. Clin. Invest. 1993. 92:2353-2359.) Key words: exercise • sympathetic nervous system • microneurography • heart failure • nuclear magnetic resonance
\end{abstract}

\section{Introduction}

During exercise, sympathetic nervous system activity is increased. This is due in part to the activation of an exercise pressor reflex (1). Animal studies suggest that the afferent limb of this reflex is composed of group III and IV neural fibers (2-4). In humans, the majority of evidence suggests that stimulation of metabolite-sensitive afferents is more important than the activation of the mechanically sensitive afferents in evoking the sympathoexcitation of exercise (5).

In a prior report, we presented evidence that the responsiveness of metabolite-sensitive afferents was reduced in heart failure (6). This conclusion was based on measurements of sympathetic nerve traffic obtained during a period of posthandgrip

Address correspondence to Dr. Lawrence I. Sinoway, Division of Cardiology, The Milton S. Hershey Medical Center, The Pennsylvania State University, P.O. Box 850, Hershey, PA 17033

Received for publication 28 January 1993 and in revised form 13 May 1993.

J. Clin. Invest.

(c) The American Society for Clinical Investigation, Inc. $0021-9738 / 93 / 11 / 2353 / 07 \quad \$ 2.00$

Volume 92, November 1993, 2353-2359 circulatory arrest (PHG-CA). ${ }^{1}$ This maneuver isolates metaboreceptor-induced sympathoexcitation from the effects of other neural systems such as muscle mechanoreceptors and central command. Interestingly, despite the fact that nerve traffic during PHG-CA was attenuated in heart failure subjects as compared with age-matched healthy controls, responses during the preceding period of static exercise were similar in the heart failure and control subjects. These findings suggest that some other neural system implicated in circulatory control during exercise must be activated to a greater degree in the heart failure subjects than in the controls.

In this report, we tested the hypothesis that increased muscle interstitial pressure, a common clinical feature seen in humans with heart failure $(7,8)$, could lead to an enhanced activation of the sympathetic nervous system via activation of muscle mechanoreceptors. The results of our experiments suggest that elevated interstitial muscle pressure sensitizes mechanoreceptors, thereby augmenting the sympathetic nervous system response to exercise.

\section{Methods}

\section{Subjects}

We studied 26 normal volunteers (mean age, 27 11 yr; range, 21-39 $\mathrm{yr}$ ). All were in good health and none were taking medications. The Hershey Medical Center Clinical Investigation Committee approved all studies, and all subjects gave informed written consent to participate.

In these experiments, we measured heart rate (HR; electrocardiogram), mean arterial blood pressure (MAP; $\mathrm{mmHg}$ ) using an automated device that uses the modified volume clamp method (Finapres; Ohmeda, Fort Lee, NJ), respiration (pneumograph), and muscle sympathetic nerve activity (MSNA) in the peroneal nerve using microneurography.

\section{Plethysmography}

Changes in forearm volume were determined using a mercury-in-silastic strain gauge $(9,10)$. Before placing the gauge on the forearm, it was externally calibrated to a tension of $10 \mathrm{~g}$. During the forearm "venous congestion" studies to be described below, the strain gauge allowed us to document the increase in forearm volume secondary to venous congestion.

\section{Microneurography}

MSNA. This technique provides direct recordings of sympathetic nerve traffic directed to blood vessels in skin or skeletal muscle $(5,11)$. This method, as used in our laboratory, has been described previously (12). Multi-unit recordings of MSNA were obtained by placing an electrode in a muscle fascicle within the peroneal nerve and a reference electrode in the adjacent subcutaneous tissue. The signal was amplified

1. Abbreviations used in this paper: $\mathrm{HR}$, heart rate; MAP, mean arterial blood pressure; MSNA, muscle sympathetic nerve activity; MVC, maximal voluntary contraction; PHG-CA, posthandgrip circulatory arrest; SSNA, skin sympathetic nerve activity. 
$(\times 50,000-90,000)$, filtered $(700$ and $2,000 \mathrm{~Hz})$, rectified, and integrated to obtain a mean voltage neurogram. The resultant neurogram was analyzed manually by counting the number of bursts and the total burst amplitude per minute (arbitrary units [U]).

Skin sympathetic nerve activity (SSNA). In pilot studies, we attempted to use a window discriminator and a counter module to count nerve spikes of SSNA. However, in some subjects, exercise increased the noise level to a value well above the voltage cutoff of the window discriminator, making data interpretation difficult. Accordingly, bursts of SSNA (and total amplitude) were counted by hand by one of the investigators in a blinded fashion. A skin burst in these studies was defined as activity greater than twice baseline noise. For complex, multi-peaked spikes, we counted each component as a separate burst if the fall in activity towards baseline was greater than one-half the amplitude of the respective component. We compared our counting method to the window discriminator method in those subjects in whom baseline noise did not increase with exercise. We found an $r$ value of 0.91 ( $P$ $<0.001$ for 96 comparisons)

\section{Protocols}

The effects of forearm venous congestion on nerve traffic during static forearm exercise. After maximal voluntary contraction (MVC) was determined, the subjects were placed supine with their nondominant forearm positioned $\sim 10 \mathrm{~cm}$ above the heart. A dynamometer was placed in the nondominant hand. The strain gauge was placed around the circumference of the mid-forearm, and an occlusion cuff was placed on the arm well above the strain gauge. The Finapres was placed on the middle finger of the opposite hand.

After $5 \mathrm{~min}$ of baseline data were collected, the upper arm cuff was inflated to $90 \mathrm{mmHg}$ for $5 \mathrm{~min}$. Prior studies in animals have suggested that venous occlusion leads to abrupt, dramatic increases in interstitial pressure (13). 5 min of "venous congestion" at $90 \mathrm{mmHg}$ was selected since this period of time and this level of upper arm cuff pressure have previously been used safely in human studies $(10,14,15)$.

The subjects performed static handgrip at $40 \%$ of MVC for $2 \mathrm{~min}$. The upper arm cuff was then inflated to $250 \mathrm{mmHg}$ and the subjects stopped exercising. PHG-CA was continued for $2 \mathrm{~min}$, and recovery data were collected for $3 \mathrm{~min}$. The subjects rested for $20 \mathrm{~min}$ and the exercise paradigm was repeated. During this second bout of exercise, a time control replaced venous occlusion preceding static exercise at $40 \%$ MVC. The sequence of the two exercise paradigms was varied.

After the subjects completed each bout of static exercise, we asked them to subjectively grade the level of forearm effort using a modified Borg scale with a range of 6-20. A value of 6 was the lowest possible effort score, and 20 represented a maximal level $(16,17)$. In an analogous manner, the subjects were asked to grade the level of forearm discomfort during the PHG-CA period that followed static handgrip. We asked them to grade this level of discomfort from 6 to 20 .

In six separate subjects, we performed the same paradigm but measured SSNA. As opposed to changes in MSNA, changes in SSNA during exercise are thought to predominantly reflect changes in central command (18-20). Thus, if the SSNA response to static exercise was greater during venous congestion than during noncongested exercise, an effect of venous congestion on central command would be implicated.

The effects of forearm venous congestion on high-energy phosphate metabolism during static forearm exercise. In five subjects, we examined the effects of forearm congestion on exercise-induced changes in high-energy phosphate metabolism. For these studies, we performed ${ }^{31} \mathrm{P}$ nuclear magnetic resonance (NMR) studies on the flexor digitorum superficialis (FDS) muscle in the forearm.

The procedures for measuring high-energy phosphate metabolites in our laboratory have been described previously (12). The ${ }^{31} \mathrm{P}$ NMR spectra were obtained with a 1.9-Tesla, 27-cm bore, superconducting magnet (Oxford Instruments, Abbington, UK) interfaced to a radiofrequency transmitter/receiver (Nicolet Instrument Corp., Madison, WI). A $2.5-\mathrm{cm}$ coil was placed over the FDS muscle in the forearm. The proton homogeneity was maximized by adjusting the room tem- perature gradients (21). The ${ }^{31} \mathrm{P}$ spectra were collected at $32.5 \mathrm{MHz}$ with a 1.9-s delay between radiofrequency pulses. The spectra that were obtained represented the Fourier transformation of 32 transients that were averaged over $60 \mathrm{~s}$.

Intracellular $\mathrm{pH}$ was calculated from the chemical shift of inorganic phosphate $(\mathrm{Pi})$ relative to the fixed peak position of phosphocreatine (PCr) (22). The relative concentrations of $\mathrm{Pi}$ and $\mathrm{PCr}$ were determined by calculating the area under their respective resonance. These concentrations were expressed in arbitrary units. The relative concentration of diprotonated phosphate was derived from the $\mathrm{pH}$, the relative concentration of total $\mathrm{Pi}$, and the $\mathrm{pKa}$ for the conversion of $\mathrm{HPO}_{4}^{-}$to $\mathrm{H}_{2} \mathrm{PO}_{4}^{-}$(23).

The effects of forearm venous congestion on nerve traffic during forearm exercise with regional circulatory arrest. In separate studies, we performed similar forearm congesting experiments except that circulatory arrest was initiated at the start of static forearm exercise. At the end of forearm exercise, we maintained circulatory arrest for $2 \mathrm{~min}$ to specifically examine metaboreceptor responses. We performed these experiments to determine the effects of venous congestion independent of any changes in blood flow that might accompany the increased interstitial pressure. At the end of these experiments, the degree of exercise-induced fatigue and forearm discomfort were evaluated as described earlier (Borg scale).

The effects of forearm venous congestion on the MSNA responses to involuntary contraction. The rationale for these studies was that MSNA responses to voluntary contraction should reflect changes in sympathetic outflow due to stimulation of muscle afferents. If venous congestion led to a greater increase in MSNA during involuntary exercise, then an effect of venous congestion on mechanically sensitive afferents would be suggested.

In six subjects, involuntary biceps contractions were performed at $\sim 25 \%$ of the predetermined MVC value. The static contractions were sustained for $1 \mathrm{~min}$, with and without forearm venous congestion.

The effects of forearm venous congestion during the cold pressor test. In six subjects, we examined the effects of forearm venous congestion on a nonspecific sympathetic stimulus, ice applied to the hand. For these studies, we placed the forearm in ice water for $90 \mathrm{~s}$ with and without first inducing forearm congestion by the 5-min venous congesting intervention.

\section{Statistics}

In these experiments, we used a two-way analysis of variance to examine the various hemodynamic and metabolic parameters. This analysis was performed to test for two main effects: stage of the paradigm and verious congestion. The simple effects were used to compare the effects of venous congestion at specific time points in the paradigm. A $P$ $<0.05$ was considered statistically significant, and all data in this report are presented as mean \pm standard error.

\section{Results}

Venous congestion increased forearm venous volume by $4.7 \pm 0.2 \%$. This was not due primarily to an increase in venous volume since forearm volume fell by only $0.9 \%$ upon the release of venous congestion. If the majority of the increase in volume were in the venous system, we would have expected a far larger immediate drop in forearm volume upon the release of PHG-CA.

The effects of forearm venous congestion on the cardiovascular responses to the static exercise paradigm. We obtained blood pressure and heart rate data in 15 subjects (Table I). Of note, we observed a venous congestion main effect and a statistical interaction for $\Delta$ blood pressure such that blood pressure was higher after venous congestion. The simple effects demonstrated that the increase in $\Delta$ blood pressure was statistically higher during the second minute of grip after forearm venous congestion. There was no statistical effect of venous congestion 
Table I. The Effects of Forearm Venous Congestion on the Cardiovascular Responses to the Nonischemic Exercise Paradigm

\begin{tabular}{|c|c|c|c|c|c|c|c|}
\hline & $\mathrm{VC} / \mathrm{TC}$ & Grip 1 & Grip 2 & PHG-CA 1 & PHG-CA 2 & REC & Main effects \\
\hline \multicolumn{8}{|l|}{$\Delta \mathrm{HR}$} \\
\hline No VC & $0.6 \pm 0.3$ & $13.8 \pm 1.7$ & $18.2 \pm 2.8$ & $1.0 \pm 1.3$ & $-0.2 \pm 1.2$ & $2.2 \pm 0.9$ & Work $F=48.4 P<0.001$ \\
\hline VC & $1.0 \pm 0.5$ & $17.3 \pm 2.0^{*}$ & $22.8 \pm 2.9^{*}$ & $2.4 \pm 1.6$ & $0.3 \pm 1.4$ & $2.0 \pm 0.6$ & $\begin{array}{l}\text { VC } F=13.2 P<0.003 \\
\text { Work } \cdot \text { VC } F=3.5 P<0.007\end{array}$ \\
\hline \multicolumn{8}{|l|}{$\triangle$ MAP } \\
\hline No VC & $-0.9 \pm 0.5$ & $11.8 \pm 1.3$ & $25.1 \pm 2.3$ & $20.0 \pm 1.9$ & $21.4 \pm 2.4$ & $5.8 \pm 1.4$ & Work $F=111.0 P<0.001$ \\
\hline VC & $1.0 \pm 0.4^{*}$ & $12.6 \pm 1.4$ & $32.7 \pm 1.7^{*}$ & $24.7 \pm 1.3$ & $25.4 \pm 1.5$ & $8.6 \pm 1.0$ & $\begin{array}{l}\text { VC } F=9.4 P<0.009 \\
\text { Work } \cdot \text { VC } F=3.2 P<0.02\end{array}$ \\
\hline \multicolumn{8}{|l|}{$\Delta$ MSNA } \\
\hline No VC & $-4.7 \pm 3.8$ & $24.8 \pm 21.0$ & $150.5 \pm 41.8$ & $162.5 \pm 45.7$ & $147.3 \pm 54.8$ & $48.6 \pm 22.0$ & Work $F=15.1 P<0.001$ \\
\hline VC & $-9.1 \pm 5.6$ & $79.9 \pm 22.0^{*}$ & $317.3 \pm 69.9^{*}$ & $232.9 \pm 51.4$ & $232.2 \pm 41.5$ & $71.2 \pm 23.6$ & $\begin{array}{l}\mathrm{VC} F=10.9 P<0.01 \\
\text { Work } \cdot \mathrm{VC} F=4.5 P<0.002\end{array}$ \\
\hline \multicolumn{8}{|l|}{$\Delta$ SSNA } \\
\hline No VC & $19.8 \pm 10.3$ & $73.7 \pm 20.1$ & $76.5 \pm 29.7$ & $32.4 \pm 27.2$ & $47.0 \pm 27.3$ & $-2.2 \pm 22.1$ & Work $F=5.0 P<0.003$ \\
\hline VC & $26.4 \pm 12.8$ & $56.7 \pm 28.4$ & $87.5 \pm 45.2$ & $22.3 \pm 33.1$ & $40.5 \pm 29.6$ & $17.5 \pm 20.2$ & $\begin{array}{l}\mathrm{VC} F=0.1 P=\mathrm{NS} \\
\text { Work } \cdot \mathrm{VC} F=1.1 P=\mathrm{NS}\end{array}$ \\
\hline
\end{tabular}

VC/TC, 5 min of either venous congestion (VC) or time control (TC); Grip 1, data from the first minute of nonischemic static handgrip at $40 \%$ MVC; Grip 2, data from the second minute of static handgrip; PHG-CA, 1 min of PHG-CA; REC, 1 min of recovery. ${ }^{*} P<0.05$ congestion vs. no congestion by simple effects. $n=15$ for $\mathrm{HR}$ (beats $/ \mathrm{min}$ ) and MAP ( $\mathrm{mm} \mathrm{Hg}$ ); $n=10$ for MSNA (U); $n=6$ for SSNA (U).

on $\Delta$ blood pressure during the 2 min of circulatory arrest. Static exercise performed during venous congestion evoked a greater HR response than exercise performed without venous congestion (Table I).

MSNA was measured in 10 subjects during the nonischemic protocol. We observed a venous congestion main effect and a statistical interaction (Table I). The simple effects showed that MSNA was higher after venous congestion during both minutes of grip. During the second minute of static handgrip, this effect was especially impressive, with the increase in $\Delta$ amplitude from baseline being twice as great after venous congestion ( $150.5 \pm 41.8$ vs. $317.3 \pm 69.9 \mathrm{U})$.

During PHG-CA, the effects of venous congestion on MSNA were unimpressive, with the exception of one subject who parenthetically was the only subject studied on two separate days. During PHG-CA without venous congestion, this subject's average $\Delta$ MSNA value was $71.1 \mathrm{U}$ over the baseline value (an $81.2 \%$ increase). During PHG-CA with venous congestion, the average $\Delta$ MSNA value was $476.4 \mathrm{U}$ (a $558.8 \%$ increase over the baseline value). In Fig. 1, we present representative neurograms from one subject.

Venous congestion increased the perceived level of effort during nonischemic exercise (13.6 \pm 0.7 vs. $17.3 \pm 0.4 \mathrm{U} ; P$ $<0.002$ ), but had no effect on the subjects' perception of forearm discomfort during the PHG-CA period (15.7 \pm 0.8 vs. $16.8 \pm 0.5 \mathrm{U} ; P=\mathrm{NS}$ ).

In the six subjects in whom we measured SSNA, we observed no effect of forearm venous congestion on $\triangle$ SSNA during exercise or during the period of PHG-CA (Table I). A similar lack of effect of venous congestion would have been noted if we had analyzed the percent change in amplitude from baseline.

The effects of forearm venous congestion on high-energy phosphate metabolism during static forearm exercise. In Table II, we present the ${ }^{31} \mathrm{P}$ NMR data obtained during the paradigm. Venous congestion had no effect on cellular $\mathrm{pH}$ or the percent change in $\mathrm{H}_{2} \mathrm{PO}_{4}^{-}$measured during the various stages of the paradigm. In addition, the $\mathrm{Pi} /(\mathrm{Pi}+\mathrm{PCr})$ ratio, an index of the amount of cellular work being performed, was not affected by forearm venous congestion. In Fig. 2, we summarize the hemodynamic and muscle metabolic responses to the static handgrip paradigm.

The effects of forearm venous congestion on the cardiovascular responses to the static exercise paradigm performed during regional circulatory arrest. In general, the results of these experiments were similar to those noted during the handgrip experiments without circulatory arrest. The results of these studies are shown in Table III. Blood pressure data were obtained in 11 subjects. We observed a venous congestion main effect. Simple effects suggested that blood pressure was higher during each stage of the protocol, including the 2 min of PHG-CA.

We obtained heart rate data in 11 subjects and demonstrated that venous congestion led to a greater increase in heart rate during the second minute of static exercise.

Venous congestion led to greater increases in MSNA during the $2 \mathrm{~min}$ of ischemic handgrip. Of note, in the nonvenous congestion portion of the paradigm, six of seven subjects had either no change ( $n=1)$ or a fall $(n=5)$ in MSNA during the first minute of handgrip as compared with baseline. After venous congestion, MSNA was higher than baseline in six of seven subjects.

As in the nonischemic experiments, venous congestion increased the perceived level of effort ( $14.2 \pm 1.0$ vs. $16.0 \pm 1.2 \mathrm{U}$; $P<0.03$ ) but had no effect on the perceived level of forearm discomfort during the PHG-CA period ( $16.0 \pm 1.0$ vs. $15.5 \pm 1.1$ $\mathrm{U} ; P=\mathrm{NS}$ ).

The effects of forearm venous congestion on the MSNA responses to involuntary contraction. Involuntary biceps static contractions performed without forearm venous congestion did not raise MSNA values above baseline. This is contrasted with the effects of involuntary contractions performed with venous congestion ( $\Delta$ MSNA $-3.0 \pm 12.3 \mathrm{U}$ during contraction 


\section{No Venous Congestion}

\section{Venous Congestion}

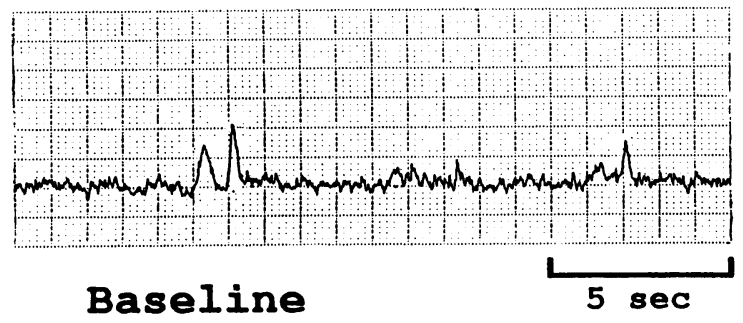

Baseline

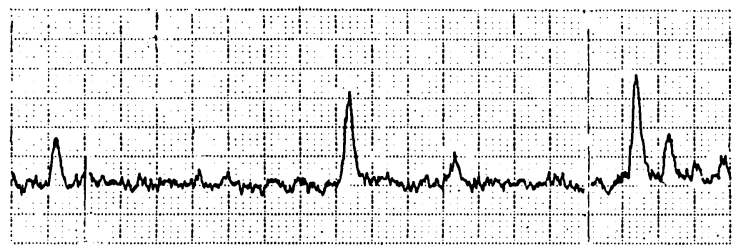

Static Exercise

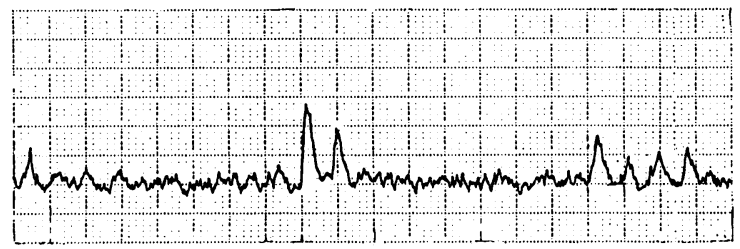

PHG-CA

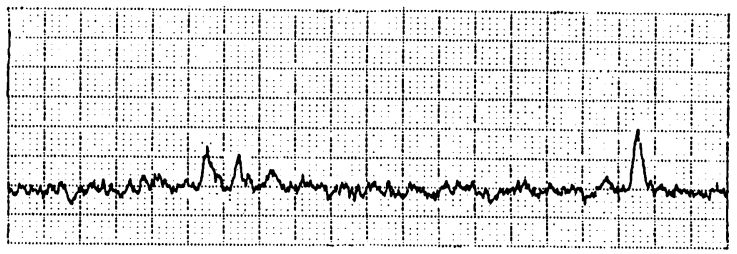

Baseline

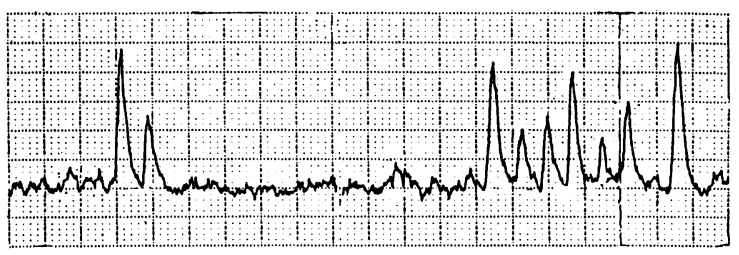

Static Exercise

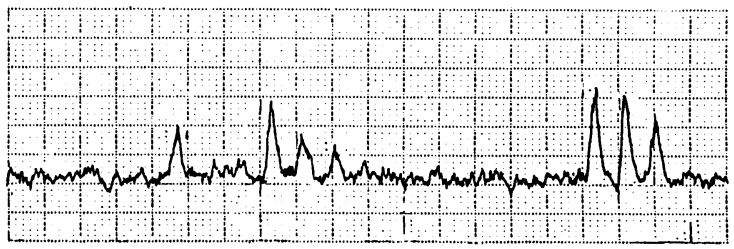

PHG-CA

Figure 1. Representative neurograms from one subject before (time control) and after venous congestion. Venous congestion augmented the MSNA response to static exercise.

without venous congestion vs. $73.3 \pm 12.5 \mathrm{U}$ during contraction with venous congestion; $P<0.001)$. The change in $\Delta$ MSNA represented a $64 \%$ increase in activity from baseline.

The effects of forearm venous congestion on the cold pressor test. Forearm venous congestion had no effect on the nerve traffic response to the cold pressor test $(152.8 \pm 32.2$ vs. 150.8 $\pm 66.7 \mathrm{U} ; P=\mathrm{NS} ; n=6)$.

\section{Discussion}

The major findings in this report are that venous congestion augmented reflex responses to static exercise and increased the perceived level of effort. In this section, we discuss the study rationale, the implications of our findings, and the limitations of our results.

Study rationale. In a prior report, we demonstrated that MSNA responses during a period of PHG-CA were attenuated in subjects with heart failure (6). Despite this, sympathetic nerve traffic was not attenuated during static exercise. Based on these observations, we hypothesized that some other neural system would need to be activated to a greater degree in heart failure subjects compared with normal control subjects. Specifi- cally, we considered the possibility that central command and/ or the augmented stimulation of mechanically sensitive afferents in skeletal muscle were responsible for those findings.

A previous report demonstrated that the sensitivity of mechanically sensitive afferents was enhanced when carrageen was injected into muscle (24). The authors of this report suggested that, aside from potential inflammatory influences, carrageen may also have increased interstitial muscle volume and pressure, and thereby lowered the threshold for mechanical stimulation of muscle afferents. In addition, work by McCloskey et al. (25) and Stebbins et al. (26) demonstrated that pressure applied directly to the triceps surae of the cat was capable of evoking pressor responses. Based on these reports, we speculated that muscle edema could act to decrease the threshold for stimulation of mechanically sensitive afferents during exercise and thereby augment the sympathetic reflex response to exercise. The results of this group of experiments seem entirely consistent with this scenario.

Forearm congestion and reflex responses. In this report, we increased forearm volume by $4.7 \%$ using a venous occlusion method. We and others have used this method previously to raise intravascular pressures above zero to test the role of myogenic influences in circulatory control $(10,14,15)$. These prior 
Table II. The Effects of Forearm Venous Congestion on High-Energy Phosphate Metabolism during Nonischemic Forearm Exercise

\begin{tabular}{|c|c|c|c|c|c|c|c|c|}
\hline & Base & $\mathrm{VC} / \mathrm{TC}$ & Grip 1 & Grip 2 & PHG-CA 1 & PHG-CA 2 & REC & Main effects \\
\hline \multicolumn{9}{|l|}{$\mathrm{pH}$} \\
\hline No VC & $7.08 \pm 0.02$ & $7.11 \pm 0.04$ & $7.02 \pm 0.01$ & $6.76 \pm 0.04$ & $6.64 \pm 0.04$ & $6.62 \pm 0.03$ & $6.76 \pm 0.06$ & Work $F=26.8 P<0.001$ \\
\hline VC & $7.11 \pm 0.03$ & $7.12 \pm 0.02$ & $7.02 \pm 0.01$ & $6.78 \pm 0.06$ & $6.67 \pm 0.07$ & $6.64 \pm 0.12$ & $6.82 \pm 0.10$ & $\begin{array}{l}\text { VC } F=0.8 P=\mathrm{NS} \\
\text { Work } \cdot \text { VC } F=0.2 P=\mathrm{NS}\end{array}$ \\
\hline \multicolumn{9}{|c|}{$\Delta \% \mathrm{H}_{2} \mathrm{PO}_{4}^{-}$} \\
\hline No VC & & $-9.1 \pm 2.3$ & $124.1 \pm 14.5$ & $360.3 \pm 26.5$ & $376.9 \pm 22.5$ & $421.3 \pm 21.1$ & $125.4 \pm 20.5$ & Work $F=80.9 P<0.001$ \\
\hline VC & & $0.9 \pm 8.0$ & $157.0 \pm 23.8$ & $393.4 \pm 76.6$ & $417.5 \pm 66.0$ & $423.8 \pm 96.6$ & $130.2 \pm 39.1$ & $\begin{array}{l}\text { VC } F=0.2 P=\mathrm{NS} \\
\text { Work } \cdot \text { VC } F=0.1 P=\text { NS }\end{array}$ \\
\hline \multicolumn{9}{|c|}{$\mathrm{Pi} /(\mathrm{Pi}+\mathrm{PCr})$} \\
\hline No VC & $0.18 \pm 0.02$ & $0.18 \pm 0.02$ & $0.39 \pm 0.04$ & $0.56 \pm 0.06$ & $0.52 \pm 0.04$ & $0.52 \pm 0.04$ & $0.26 \pm 0.03$ & Work $F=52.1 P<0.001$ \\
\hline vC & $0.18 \pm 0.02$ & $0.19 \pm 0.02$ & $0.41 \pm 0.04$ & $0.55 \pm 0.08$ & $0.55 \pm 0.07$ & $0.52 \pm 0.07$ & $0.27 \pm 0.03$ & $\begin{array}{l}\mathrm{VC} F=1.0 P=\mathrm{NS} \\
\text { Work } \cdot \mathrm{VC} F=0.5 P=\mathrm{NS}\end{array}$ \\
\hline
\end{tabular}

Base, 5 min of baseline; VC/TC, 5 min of either venous congestion (VC) or time control (TC); Grip, 1 min of nonischemic static handgrip at $40 \%$ MVC; PHG-CA, 1 min of PHG-CA; REC, 1 min of recovery. $n=5$.

experiments suggest that this intervention is well tolerated and does not cause forearm venous thrombosis. In addition, studies by Guyton (13) suggest that raising venous pressure in a manner similar to the method used in this study will substantially raise interstitial pressure almost immediately.

In these studies, the MSNA response to static exercise was augmented when muscle interstitial pressure was elevated. This effect was not predominantly due to greater stimulation of muscle metaboreceptors since MSNA was not higher during the PHG-CA phase of the paradigm. Moreover, as demonstrated in our ischemic forearm exercise paradigm, elevated interstitial pressure augmented cardiovascular reflex responses during forearm exercise in the absence of blood flow. Thus, elevated interstitial pressure did not exert its effect by reducing blood flow to exercising muscle. Our studies examining MSNA responses to involuntary biceps contractions suggest that ve- nous congestion augments local muscle afferent responses. Additionally, our studies examining the effects of venous congestion on skin sympathetic nerve responses suggest that central command was not increased by venous congestion. Thus, these separate sets of experiments, when viewed together, suggest that elevated interstitial edema must be stimulating nonmetabolite-sensitive afferents in the exercising skeletal muscle. Since group I and II muscle afferent fibers did not mediate cardiovascular reflex responses in animals (25), we have concluded that elevated interstitial pressure and volume must be sensitizing mechanically sensitive thin fiber muscle afferents.

These findings may be especially important in heart failure, where venous pressures are chronically elevated and peripheral edema is a common clinical finding. This may enhance the activation of mechanically sensitive afferents and importantly contribute to the degree of sympathoexcitation during exercise
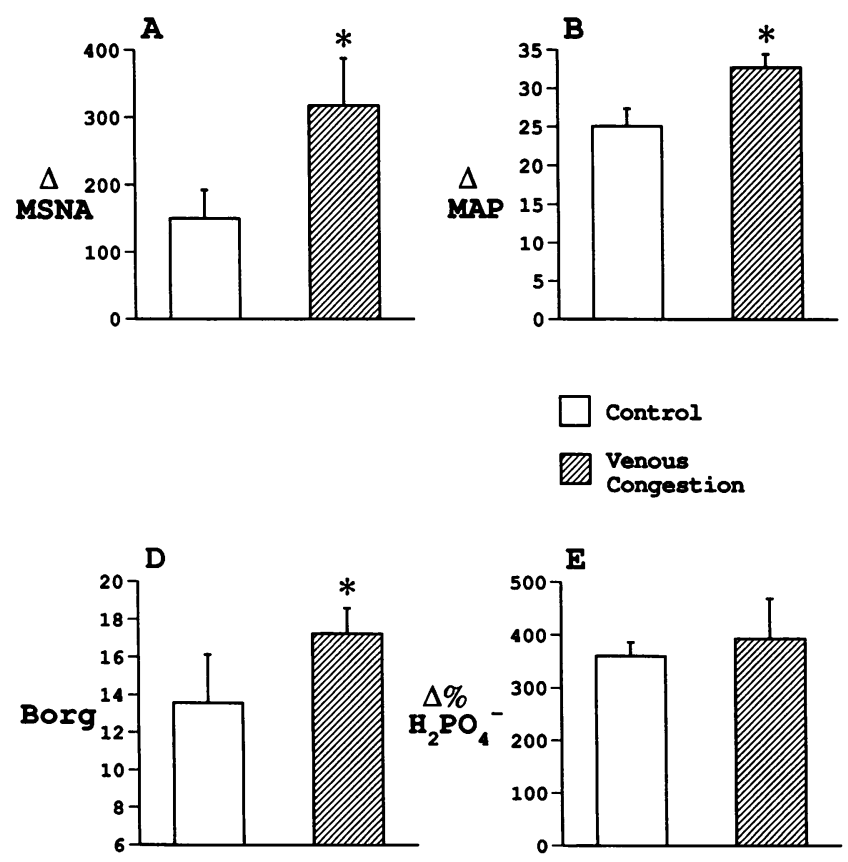

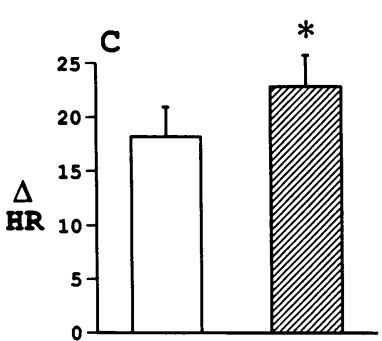

Figure 2. Reflex and muscle metabolic responses during the second minute of static exercise. The bar graphs present the mean values for: $(A) \triangle$ MSNA in arbitrary units $(n$ $=10) ;(B) \Delta$ MAP in $\mathrm{mmHg}(n$ $=15) ;(C) \Delta \mathrm{HR}$ in beats per minute $(n=15) ;(D)$ Borg scale $(n$ $=12) ;(E)$ the percent change from baseline in $\mathrm{H}_{2} \mathrm{PO}_{4}^{-}(n=5)$; and $(F)$ $\mathrm{H}^{+}$concentration in $\mathrm{nM}(n=5)$. * Statistically significant differences between control conditions (open columns) and venous congestion (hatched columns). Bars above the columns represent the standard error. 
Table III. The Effects of Forearm Venous Congestion on the Cardiovascular Responses to the Ischemic Exercise Paradigm

\begin{tabular}{|c|c|c|c|c|c|c|c|}
\hline & $\mathrm{VC} / \mathrm{TC}$ & Grip 1 & Grip 2 & PHG-CA 1 & PHG-CA 2 & REC & Main effects \\
\hline \multicolumn{8}{|l|}{$\Delta \mathrm{HR}$} \\
\hline No VC & $0.8 \pm 0.6$ & $11.9 \pm 2.2$ & $15.6 \pm 2.5$ & $3.8 \pm 2.6$ & $3.9 \pm 2.4$ & $2.0 \pm 1.1$ & Work $F=26.1 P<0.001$ \\
\hline vC & $0.4 \pm 0.3$ & $15.6 \pm 1.6$ & $21.1 \pm 3.4^{*}$ & $3.7 \pm 2.2$ & $2.0 \pm 2.0$ & $0.5 \pm 1.8$ & $\begin{array}{l}\mathrm{VC} F=0.4 P=\mathrm{NS} \\
\text { Work } \cdot \mathrm{VC} F=3.9 P<0.005\end{array}$ \\
\hline \multicolumn{8}{|l|}{$\Delta$ MAP } \\
\hline No VC & $-1.1 \pm 0.7$ & $10.3 \pm 3.0$ & $27.0 \pm 3.1$ & $21.8 \pm 2.5$ & $22.8 \pm 2.7$ & $5.7 \pm 1.4$ & Work $F=41.5 P<0.001$ \\
\hline VC & $2.8 \pm 0.8^{*}$ & $18.2 \pm 3.4^{*}$ & $36.8 \pm 4.8^{*}$ & $28.0 \pm 3.4^{*}$ & $28.0 \pm 3.2^{*}$ & $10.0 \pm 2.7$ & $\begin{array}{l}\text { VC } F=21.8 P<0.001 \\
\text { Work } \cdot \text { VC } F=1.3 P=\mathrm{NS}\end{array}$ \\
\hline \multicolumn{8}{|l|}{$\Delta$ MSNA } \\
\hline No VC & $-1.4 \pm 9.4$ & $-24.1 \pm 15.2$ & $115.2 \pm 26.1$ & $73.9 \pm 32.3$ & $74.6 \pm 37.3$ & $-7.1 \pm 17.8$ & Work $F=8.2 P<0.001$ \\
\hline VC & $-7.2 \pm 8.3$ & $40.2 \pm 14.3$ & $204.3 \pm 53.3^{*}$ & $120.4 \pm 47.2$ & $127.0 \pm 47.7$ & $30.8 \pm 18.6$ & $\begin{array}{l}\mathrm{VC} F=20.9 P<0.004 \\
\text { Work } \cdot \mathrm{VC} F=2.2 P=\mathrm{NS}\end{array}$ \\
\hline
\end{tabular}

VC/TC, 5 min of either venous congestion (VC) or time control (TC); Grip, 1 min of ischemic static handgrip at 40\% MVC; PHG-CA, 1 min of PHG-CA; REC, 1 min of recovery. ${ }^{*} P<0.05$ congestion vs. no congestion by simple effects. $n=11$ for HR and MAP; $n=7$ for MSNA. Units as in text and Table $I$.

in heart failure. Further studies will be necessary to confirm this postulate.

A surprising feature of these experiments was the greater HR response to exercise after the venous congesting maneuver. Changes in HR during forearm exercise in humans have been thought to be due to changes in central command (5). We considered the possibility that the increase in $\mathrm{HR}$ was due to a reduction in blood flow secondary to venous congestion. However, the ischemic handgrip protocol makes this possibility unlikely since venous congestion increased HR more than exercise without venous congestion.

We also considered the possibility that the greater HR response after forearm venous congestion was due to a greater level of exertion. However, our metabolic index of work, the $\mathrm{Pi} /(\mathrm{Pi}+\mathrm{PCr})$ ratio, was not affected by venous congestion. Moreover, the level of tension developed during handgrip was not greater after forearm venous congestion.

We conclude that the HR response to exercise is modified by sensory feedback emanating from exercising muscle. We speculate that this sensory information is transduced by mechanically sensitive group III and IV fibers, and this acts to either directly increase HR or modulate the effects of central command.

These observations are consistent with the work of Mitchell et al. (27), who demonstrated that the HR and blood pressure responses to static exercise at a given level of relative force were reduced by epidural anesthesia. From these results, the authors concluded that under the conditions imposed by their paradigm, sensory feedback from muscle was necessary for the full expression of central command.

Our conclusions and those of Mitchell et al. are at odds with prior experiments that used neuromuscular blockade as a method to dissociate central command from peripheral feedback emanating from the exercising skeletal muscle. These studies have suggested that HR is controlled by central command, whereas MSNA is regulated by chemically sensitive skeletal muscle afferents (18). We cannot entirely explain these different conclusions except to agree with Mitchell et al. (27) that both central and peripheral neural mechanisms may contribute to the cardiovascular reflex response to exercise.

Forearm congestion and perceived effort. Although the primary focus of this report was not to examine the causes of fatigue, we were impressed with the comments of the first few subjects, who described a greater sense of effort when exercise was performed during venous congestion. Accordingly, we asked the subjects to rate their level of perceived effort at the end of the $2 \mathrm{~min}$ of static forearm exercise using a scale modeled after the Borg range. These results demonstrate that forearm congestion increased the perception of effort. This was not a blood flow phenomenon since we observed similar results during ischemic exercise, nor was it an effect of sequence since the order of study (i.e., handgrip with venous congestion and handgrip without venous congestion) was varied.

We also asked the subjects to compare the degree of discomfort during the PHG-CA portion of the experiment. During this portion of the paradigm, only metabolite-sensitive muscle afferents should be stimulated. We observed no effect of venous congestion. Based on these results, we would suggest that increased interstitial pressure enhanced the responsiveness of the mechanically sensitive muscle afferents; and these afferents transmitted reflex information to central neural centers, where the perception of effort was sensed.

The NMR studies suggest that the increased perception of effort was not due to a greater level of metabolic work since $\mathrm{Pi} /(\mathrm{Pi}+\mathrm{PCr})$ levels were unchanged by venous congestion. Moreover, these studies suggest that the site of fatigue was not likely to be the muscle cell itself since $\mathrm{pH}$ and $\mathrm{H}_{2} \mathrm{PO}_{4}^{-}$, two important cellular determinants of muscle fatigue, were unaffected by venous congestion (28).

In short, we believe these studies are consistent with the theory that muscle afferents play a crucial role in determining perceived muscle effort (29). We should also point out that this report provides the first evidence that peripheral feedback from skeletal muscle may modulate both reflexes and the perception of effort.

Again, we believe these findings may have relevance to subjects with heart failure. These subjects complain of chronic fatigue and exercise intolerance. It has been our impression that the degree of fatigue worsens when these individuals are edematous. We would speculate that the increased stimulation of mechanically sensitive muscle afferents contributes to the sensation of fatigue and the degree of sympathoexcitation seen in these subjects. Further experiments in these subjects will be necessary to confirm this hypothesis. 
Limitations. First, we have no way of knowing how much interstitial pressure rose. We considered experiments where thin-gauge needles would be inserted into muscle to measure pressure. However, previous studies by Guyton (13) suggest that this approach is unlikely to accurately reflect interstitial pressure.

Based on our exercise studies, we could not exclude that the increased neural responses seen after venous congestion were not due to an effect of interstitial edema on skin afferents that acted to increase neural responsiveness. We performed additional cold pressor studies that demonstrated that localized interstitial edema did not augment MSNA. Since the response to this maneuver is likely to be mediated by thin fiber afferents in skin, we must conclude that the effects of increased interstitial edema cannot be generalized to all populations of thin fiber afferents.

We observed a greater increase in PHG-CA blood pressure during the ischemic exercise protocol. This suggests that metabolite-sensitive afferents were stimulated to a greater degree during venous congestion. Thus, ischemia and elevated interstitial pressure may have acted synergistically to sensitize metabolitesensitive muscle afferents. However, if stimulation of metabolite-sensitive afferents were the primary explanation for our observations, we would have expected a far more prominent effect of venous congestion on MSNA during the PHG-CA portions of both exercise paradigms.

Our findings disagree with those of Baum et al. (30). These authors demonstrated that venous occlusion of the calf did not augment the blood pressure or HR response during $5 \mathrm{~min}$ of a paradigm that simulated repetitive ischemic toe-raising exercise. In these prior experiments, the overall rise in blood pressure was small and the precise percentage of $\mathrm{VO}_{2 \max }$ (or MVC) used for the exercise and the increase in limb volume due to venous occlusion were not determined. Accordingly, direct comparison with the present report is difficult.

Finally, there are limitations to using acute venous congestion as a model for heart failure. As mentioned earlier, we cannot quantify interstitial pressure; and accordingly, we cannot compare the stimulus used in this study to conditions seen in heart failure. Second, heart failure is a chronic condition, whereas our intervention led to an acute change in interstitial volume and pressure. Additional studies will be necessary to determine the effects of chronic elevations in muscle interstitial pressure on muscle reflexes.

In conclusion, these studies provide evidence that elevated interstitial muscle pressure stimulates mechanically sensitive muscle afferents. Activation of these afferents augments the cardiovascular reflex response to exercise and increases the perceived degree of effort.

\section{Acknowledgments}

We thank Kristen Gray, Lynette Forero, and Sandra Whisler for expert lab assistance and typing of the manuscript.

This work was supported in part by the BRSG grant S07 RR05680-22 awarded by the Biomedical Research Support Grant Program, Division of Research Resources, National Institutes of Health. This work was also supported by grant HL-44667 (L. Sinoway). L. Sinoway is an Established Investigator of the American Heart Association. J. McClain was a Fellow under the Stanley J. Sarnoff Endowment for Cardiovascular Science.

\section{References}

1. Alam, M., and F. Smirk. 1937. Observations in man upon a blood pressure raising reflex arising from the voluntary muscles. J. Physiol. 89:372-383.
2. McCloskey, D., and J. Mitchell. 1972. Reflex cardiovascular and respiratory responses originating in exercising muscle. J. Physiol. 224:173-186.

3. Mitchell, J., and R. Schmidt. 1983. Handbook of Physiology. Vol. III, part 2. Waverly Press, Inc., Baltimore. 623-658.

4. Kaufman, M. P., J. C. Longhurst, K. J. Rybicki, J. H. Wallach, and J. H. Mitchell. 1983. Effects of static muscular contraction on impulse activity of group III and IV afferents in cats. J. Appl. Physiol. 55:105-112.

5. Mark, A. L., R. G. Victor, C. Nerhed, and B. G. Wallin. 1985. Microneurographic studies of the mechanisms of sympathetic nerve responses to static exercise in humans. Circ. Res. 57:461-469.

6. Sterns, D. A., S. M. Ettinger, K. S. Gray, S. K. Whisler, T. J. Mosher, M. B. Smith, and L. I. Sinoway. 1991. Skeletal muscle metaboreceptor exercise responses are attenuated in heart failure. Circulation. 84:2034-2039.

7. Braunwald, E., and W. Grossman. 1992. Clinical aspects of heart failure. In Heart Disease. A Textbook of Cardiovascular Medicine. E. Braunwald, editor. W. B. Saunders Company, Philadelphia. 444-463.

8. Sinoway, L. I., J. R. Minotti, D. Davis, J. L. Pennock, J. E. Burg, T. I. Musch, and R. Zelis. 1988. Delayed reversal of impaired vasodilation in congestive heart failure after heart transplantation. Am. J. Cardiol. 61:1076-1079.

9. Sinoway, L. I., T. Musch, D. Goldner, J. Minotti, and R. Zelis. 1987. Diuretic therapy improves maximal metabolic vasodilation in congestive heart failure. Am. J. Cardiol. 60:107-111.

10. Sinoway, L. I., J. S. Wilson, R. Zelis, J. Shenberger, D. P. McLaughlin, D. L. Morris, and F. P. Day. 1988. Sympathetic tone affects human limb vascular resistance during a maximal metabolic stimulus. Am. J. Physiol. 255 (Heart Circ. Physiol. 24): H937-H946.

11. Vallbo, A. B., K. E. Hagbarth, H. E. Torebjork, and B. G. Wallin. 1979. Somatosensory proprioceptive, and sympathetic activity in human peripheral nerves. Physiol. Rev. 59:919-956.

12. Sinoway, L. I., R. F. Rea, T. J. Mosher, M. B. Smith, and A. L. Mark. 1992. Hydrogen ion concentration is not the sole determinant of muscle metaboreceptor responses in humans. J. Clin. Invest. 89:1875-1884.

13. Guyton, A. C. 1963. A concept of negative interstitial pressure based on pressure in implanted perforated capsules. Circ. Res. 12:399-414.

14. Wood, J. E., J. Litter, and R. W. Wilkins. 1955. The mechanism of limb segment reactive hyperemia in man. Circ. Res. 3:581-587.

15. Zelis, R., D. T. Mason, and E. Braunwald. 1968. A comparison of peripheral resistance vessels in normal subjects and in patients with congestive heart failure. J. Clin. Invest. 47:960-969.

16. Borg, G. A. V. 1982. Psychophysical bases of perceived exertion. Med. Sci. Sports Exercise. 14(5):377-381.

17. Borg, G. 1970. Perceived exertion as an indicator of somatic stress. Scand. J. Rehabil. Med. 2:92-98.

18. Victor, R. G., S. L. Pryor, N. H. Secher, and J. H. Mitchell. 1989. Effects of partial neuromuscular blockade on sympathetic nerve responses to static exercise in humans. Circ. Res. 65:468-476.

19. Saito, M., M. Naito, and T. Mano. 1990. Different responses in skin and muscle sympathetic nerve activity to static muscle contraction. J. Appl. Physiol. 69(6):2085-2090.

20. Vissing, S. F., U. Scherrer, and R. G. Victor. 1991. Stimulation of skin sympathetic nerve discharge by central command. Differential control of sympathetic outflow to skin and skeletal muscle during static exercise. Circ. Res. 69:228-238.

21. Gordon, R. E., and W. E. Timms. 1982. An improved tune and match circuit for $\mathrm{B}_{\mathrm{o}}$ shimming in intact biological samples. J. Magn. Reson. 46:322324.

22. Moon, R. B., and J. H. Richards. 1973. Determination of intracellular pH by ${ }^{31} \mathrm{P}$ magnetic resonance. J. Biol. Chem. 248:7276-7278.

23. Wilson, J., K. McCully, D. Mancini, B. Boden, and B. Chance. 1988. Relationship of muscular fatigue to $\mathrm{pH}$ and diprotonated $\mathrm{Pi}$ in humans: $\mathrm{a}^{31} \mathrm{P}$ NMR study. J. Appl. Physiol. 64:2333-2339.

24. Berberich, P., U. Hoheisel, and S. Mense. 1988. Effects of a carrageenaninduced myositis on the discharge properties of group III and IV muscle receptors in the cat. J. Neurophysiol. 59:1395-1409.

25. McCloskey, D. I., P. B. C. Matthews, and J. H. Mitchell. 1972. Absence of appreciable cardiovascular and respiratory responses to muscle vibration. $J$. Appl. Physiol. 33(5):623-626.

26. Stebbins, C. L., B. Brown, D. Levin, and J. C. Longhurst. 1988. Reflex effect of skeletal muscle mechanoreceptor stimulation on the cardiovascular system. J. Appl. Physiol. 65(4):1539-1547.

27. Mitchell, J. H., D. R. Reeves, Jr., H. B. Rogers, and N. H. Secher. 1989. Epidural anaesthesia and cardiovascular responses to static exercise in man. $J$. Physiol. 417:13-24.

28. Nosek, T., K. Fender, and R. Godt. 1987. It is diprotonated inorganic phosphate that depresses force in skinned skeletal muscle fibers. Science (Wash. DC). 236:191-192.

29. Bigland-Ritchie, B., N. J. Dawson, R. S. Johansson, and O. C. J. Lippold. 1986. Reflex origin for the slowing of motorneurone firing rates in fatigue of human voluntary contractions. J. Physiol. (Lond.). 379:451-459.

30. Baum, K., D. Essfeld, and J. Stegemann. 1990. Reduction in extracellular muscle volume increases heart rate and blood pressure response to isometric exercise. Eur. J. Appl. Physiol. Occup. Physiol. 60:217-221. 